\title{
The checklist of the macromycetes determined in Gaziantep province
}

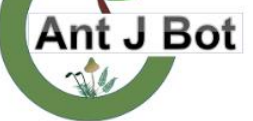

Received : 09.06.2020

Accepted : 09.07.2020 Online : 26.10 .2020
Yasin UZUN ${ }^{1 *}$, Abdullah KAYA ${ }^{2} \odot$

${ }^{1}$ Karamanoğlu Mehmetbey University, Science Faculty, Department of Biology, Karaman, Turkey

${ }^{2}$ Gazi University, Science Faculty, Department of Biology, Ankara, Turkey

*yuclathrus@gmail.com, ${ }^{2}$ kayaabd@hotmail.com

\section{Gaziantep yöresinde belirlenen makromantarların kontrol listesi}

Abstract: This study was based on the findings of macrofungal studies performed within the boundaries of Gaziantep Province between 2009 and 2019. Tracing the researches carried out in the region, a list of 404 taxa belonging to 203 genera, 80 families, 23 order and 8 classes within Ascomycota and Basidiomycota have been compiled.

Key words: Biodiversity, macrofungi, Turkey

Özet: Bu çalışma 2009 ve 2019 yılları arasında Gaziantep il sınırları içinde makromantarlar üzerinde gerçekleştirilmiş çalışmaların bulgularına dayanmaktadır. Bölgede gerçekleştirilen çalışmalar taranarak Ascomycota ve Basidiomycota bölümleri içinde yer alan 8 sınıf, 23 takım, 80 familya ve 203 cinse ait 404 taksonu içeren bir liste oluşturulmuştur.

Anahtar Kelimeler: Biyoçeşitlilik, makromantarlar, Türkiye

Citation: Uzun Y, Kaya A (2020). The checklist of the macromycetes determined in Gaziantep province. Anatolian Journal of Botany 4(2): 106-115.

\section{Introduction}

There has been an increase in the number of taxonomic studies on Turkish macromycota, especially in the last three to four decades. The latest checklists on the macrofungi of Turkey (Sesli and Denchev, 2014; Solak et al., 2015) contain about 2400 macromycete taxa, three of which were presented as new species (Intini et al., 2003; Işıloğlu et al., 2009, 2010; Watling et al., 2010). Following the checklists, some local lists (Demirel and Koçak, 2016; Güngör et al., 2016; Demirel et al., 2017; Kaşık et al., 2017; Türkekul and Işık, 2017, 2019; Uzun et al., 2017; Demirel and All1, 2019; Y1ld1z et al., 2019; Sadullahoğlu and Uzun, 2020; Uzun et al., 2020), some new species (Sesli et al., 2015, 2017, 2018; Vizzini et al., 2015, 2016; Taşkın et al., 2016; Sesli and Vizzini, 2017; Doğan et al., 2018; Kaygusuz et al., 2020) and many new records (Acar and Uzun, 2017; Çolak et al., 2017; Topçu Sesli and Sesli, 2017; Akata et al., 2018; Doğan et al., 2018; Işık and Türkekul, 2018; Sesli et al., 2018; Akçay, 2019, 2020; Keleş, 2019; Şen and Allı, 2019; Işık, 2020) were also presented.

Gaziantep is a province of Turkey within Southeastern Anatolian region and is among the studied regions of Turkey. In Gaziantep, the first mycological study related to macrofungi was carried out by Kaya (2009) in a restricted area (Huzurlu High Plateau). Starting from 2012, three local lists from Araban (Kaya et al. 2012), Şehitkamil and Yavuzeli (Kaya et al. 2014), and İslahiye (Uzun et al. 2015a) districts were presented. Following these studies 83 macromycete taxa have been reported as new records for the macromycota of Turkey from the region (Karacan et al., 2015, Kaya and Uzun, 2015, 2018; Kaya et al., 2015, 2016, 2018; Uzun et al., 2015b; Uzun et al., 2016; Uzun et al., 2017a,b,c; Uzun et al., 2018a,b,c). Lastly a contributory list was also presented about the macromycete taxa of the region by Kaya et al. (2019).

The current study was based on the findings of the studies that have so far been carried out within the boundaries of
Gaziantep province. The aim of the study is to present the marcromycetes of Gaziantep province as a complete list and to contribute to the knowledge of the mycobiota of Turkey.

\section{Materials and Method}

The researches published on macromycetes of Gaziantep province were traced and a list of the macromycete taxa was prepared together with the references they were presented in. During preparation of the list, only the taxa presented in a peer reviewed article were considered, and those presented in conference papers, graduate theses or project reports were not included. The authors names of fungal taxa are abbreviated according to Kirk \& Ansell (1992) and Kirk et al. (2004). The systematic of the taxa follows Cannon and Kirk (2007), Kirk et al. (2008), and Index fungorum (accessed 20 December 2019).

\section{Results}

The list of the taxa, reported from the region within the boundaries of Gaziantep province are given in alphabetical order together with the references they were presented in.

Fungi R.T. Moore

Ascomycota Caval.-Sm.

Dothideomycetes O.E. Erikss. \& Winka

Patellariales D. Hawksw. \& O.E. Erikss.

Patellariaceae Corda

1. Patellaria atrata (Hedw.) Fr.: (Kaya et al., 2019).

Leotiomycetes O.E. Erikss. \& Winka

Helotiales Nannf.

Dermateaceae Fr.

2. Mollisia cinerea (Batsch) P. Karst.: (Hedw.) Fr.: (Kaya et al., 2015).

3. Mollisia hydrophila (P. Karst.) Sacc.: (Hedw.) Fr.: (Kaya et al., 2019).

4. Mollisia ligni (Desm.) P. Karst.: (Hedw.) Fr.: (Kaya et al., 2019). 
5. Mollisia melaleuca (Fr.) Sacc.: (Hedw.) Fr.: (Kaya et al., 2019).

6. Tapesia fusca (Pers.) Fuckel: (Uzun et al., 2015a).

7. Tapesia strobilicola (Rehm) Sacc.: (Uzun et al., 2015a).

8. Trichobelonium kneiffii (Wallr.) J. Schröt.: (Hedw.) Fr.: (Kaya et al., 2015).

Helotiaceae Rehm

9. Ascocoryne cylichnium (Tul.) Korf: (Kaya et al., 2019).

10. Bisporella citrina (Batsch) Korf \& S.E. Carp.: (Uzun et al., 2015a).

11. Bisporella sulfurina (Quél.) S.E. Carp.: (Kaya et al., 2018).

12. Cenangium ferruginosum Fr.: (Kaya et al., 2019).

13. Hymenoscyphus calyculus (Fr.) W. Phillips: (Kaya et al., 2019).

14. Hymenoscyphus fructigenus (Bull.) Gray: (Kaya et al., 2019).

15. Hymenoscyphus herbarum (Pers.) Dennis: (Kaya et al., 2019).

16. Hymenoscyphus janthinum (Fr.) Lambotte: (Uzun et al., 2015a).

17. Hymenoscyphus scutula (Pers.) W. Phillips: (Uzun et al., 2015a).

18. Hymenoscyphus serotinus (Pers.) W. Phillips: (Kaya et al., 2019).

19. Phaeohelotium umbilicatum (Le Gal) Dennis: (Kaya, 2009).

Hyaloscyphaceae Nannf.

20. Calycina conorum (Rehm) Baral: (Uzun et al., 2017d).

21. Dasyscyphella nivea (R. Hedw.) Raitv.: (Uzun et al., 2015a).

22. Discocistella grevillei (Berk.) Svrček: (Uzun et al., 2017d).

23. Hyalopeziza millepunctata (Lib.) Raitv.: (Uzun et al., 2017d).

24. Lachnellula subtilissima (Cooke) Dennis: (Uzun et al., 2015a).

25. Lasiobelonium horridulum var. capitatum Dougoud: (Kaya et al., 2015).

26. Lasiobelonium variegatum (Fuckel) Raitv.: (Uzun et al., 2017c).

27. Perrotia flammea (Alb. \& Schwein.) Boud.: (Uzun et al., 2015a).

28. Rodwayella citrinula (P. Karst.) Spooner: (Uzun et al., 2017d).

Lachnaceae Raitv.

29. Lachnum fuscescens (Pers.) P. Karst.: (Kaya et al., 2018).

30. Lachnum virgineum (Batsch) P. Karst.: (Uzun et al., 2015a).

31. Neobulgaria pura (Pers.) Petr.: (Uzun et al., 2015b).

32. Trichopeziza subsulphurea (Svrček) Baral: (Uzun et al., 2015b).

Marthamycetaceae H.O. Baral, G. Lantz, Hustad \&

Minter

33. Cyclaneusma minus (Butin) DiCosmo, Peredo \& Minter: (Kaya et al., 2014).

34. Cyclaneusma niveum (Pers.) DiCosmo, Peredo \& Minter: (Kaya et al. 2018).

35. Naemacyclus fimbriatus (Schwein.) DiCosmo, Peredo \& Minter: (Kaya et al., 2019).
Rutstroemiaceae Holst-Jensen

36. Rutstroemia firma (Pers.) P. Karst.: (Kaya et al., 2019).

Sclerotiniaceae Whetzel

37. Ciboria rufofusca (O. Weberb.) Sacc.: (Kaya et al., 2019).

38. Sclerotinia sclerotiorum (Lib.) de Bary: (Kaya et al., 2019).

39. Sclerotinia trifoliorum Erikss.: (Kaya and Uzun, 2018).

Rhytismatales M.E. Barr ex Minter

Rhytismataceae Chevall.

40. Coccomyces delta (Kunze ex Fr.) Sacc.: (Kaya and Uzun, 2018).

41. Coccomyces dentatus (J.C. Schmidt) Sacc.: (Kaya and Uzun, 2018).

42. Lophodermium arundinaceum (Schrad.) Chevall.: (Kaya et al., 2019).

43. Propolis farinosa (Pers.) Fr.: (Kaya et al., 2019).

Orbiliomycetes O.E. Erikss. \& Baral

Orbiliales Baral, O.E. Erikss.

Orbiliaceae Nannf

44. Hyalorbilia inflatula (P. Karst.) Baral \& G. Marson: (Kaya et al. 2018).

45. Orbilia aristata (Velen.) Velen.: (Kaya et al., 2018).

Pezizomycetes O.E. Erikss. \& Winka

Pezizales J. Schröt.

Ascobolaceae Boud. ex Sacc.

46. Ascobolus carbonarius P. Karst.: (Uzun et al., 2018b). 47. Ascobolus crenulatus P. Karst.: (Uzun et al., 2018b).

48. Ascobolus foliicola Berk. \& Broome: (Uzun et al., 2018b).

49. Ascobolus immersus Pers.: (Uzun et al. 2018b).

50. Ascobolus stercorarius (Bull.) J. Schröt.: (Kaya et al., 2014).

51. Saccobolus glaber (Pers.) Lambotte: (Uzun et al., 2018b).

52. Thecotheus holmskioldii (E.C. Hansen) Eckblad: (Uzun et al., 2018b).

53. Thecotheus pelletieri (P. Crouan \& H. Crouan) Boud.: (Kaya and Uzun, 2015).

Ascodesmidaceae J. Schröt.

54. Lasiobolus cuniculi Velen.: (Uzun et al., 2018b).

Caloscyphaceae Harmaja

55. Caloscypha fulgens (Pers.) Boud.: (Kaya et al., 2019).

Helvellaceae Fr.

56. Barssia hellenica Kaounas, Agnello, P. Alvarado \& Slavova: (Uzun et al., 2018b).

57. Helvella acetabulum (L.) Quél.: (Kaya et al., 2019).

58. Helvella compressa (Snyder) N.S. Weber: (Uzun et al., 2015a).

59. Helvella costifera Nannf.: (Uzun et al., 2015a).

60. Helvella dissingii Korf: (Kaya et al., 2019).

61. Helvella lacunosa Afzel.: (Kaya et al., 2019).

62. Helvella leucomelaena (Pers.) Nannf.: (Kaya et al., 2019).

63. Helvella leucopus Pers.: (Kaya et al., 2019).

64. Helvella macropus (Pers.) P. Karst.: (Kaya et al., 2019).

65. Helvella monachella (Scop.) Fr.: (Kaya et al., 2019). 66. Paxina queletii (Bresadola) Stangl: (Kaya et al., 2019). 
Morchellaceae Rchb.

67. Mitrophora semilibera (DC.) Lév.: (Kaya et al., 2019).

68. Morchella deliciosa Fr.: (Kaya et al., 2019).

69. Morchella elata Fr.: (Kaya et al., 2019).

70. Morchella esculenta (L.) Pers.: (Kaya et al., 2019).

71. Verpa conica (O.F. Müll.) Sw.: (Kaya et al., 2019).

Pezizaceae Dumort.

72. Iodophanus carneus (Pers.) Korf: (Kaya et al., 2019).

73. Marcelleina atroviolacea Brumm.: (Uzun et al., 2018b).

74. Marcelleina rickii (Rehm) Graddon: (Uzun et al., 2018b).

75. Peziza badia Pers.: (Kaya et al., 2019).

76. Peziza cerea Sowerby ex Fr.: (Kaya et al., 2019).

77. Peziza fimeti (Fuckel) E.C. Hansen: (Kaya et al., 2019).

78. Peziza pseudoviolacea Donadini: (Kaya et al., 2019).

79. Peziza vesiculosa Bull.: (Kaya et al., 2019).

80. Peziza violacea Pers.: (Kaya et al., 2019).

81. Sarcosphaera coronaria (Jacq.) J. Schröt.: (Kaya et al., 2019).

82. Terfezia boudieri Chatin: (Kaya et al., 2019).

83. Terfezia olbiensis Tul. \& C. Tul.: (Uzun et al., 2015a).

Pyronemataceae Corda

84. Aleuria exigua Rifai: (Kaya et al. 2016).

85. Cheilymenia catenipila J. Moravec: (Kaya et al., 2016).

86. Cheilymenia fimicola (Bagl.) Dennis: (Kaya et al., 2019).

87. Cheilymenia pulcherrima (P. Crouan \& H. Crouan) Boud.: (Uzun et al., 2018b).

88. Cheilymenia theleboloides (Alb. \& Schwein.) Boud.: (Kaya et al., 2019).

89. Cheilymenia vitellina (Pers.) Dennis: (Kaya et al., 2016).

90. Geopora arenicola (Lév.) Kers: (Kaya et al., 2019).

91. Geopora arenosa (Fuckel) S. Ahmad: (Kaya et al., 2019).

92. Geopora sumneriana (Cooke) M. Torre: (Kaya et al., 2019).

93. Geopyxis majalis (Fr.) Sacc.: (Kaya et al., 2016).

94. Geopyxis vulcanalis (Peck) Sacc.: (Kaya et al., 2016).

95. Humaria aurantia (Clem.) Häffner, Benkert \& Krisai: (Kaya et al., 2016).

96. Humaria hemisphaerica (F.H. Wigg.) Fuckel: (Kaya et al., 2019).

97. Hypotarzetta insignis (Berthet \& Riousset) Donadini: (Kaya and Uzun, 2015; Kaya et al., 2019).

98. Inermisia gyalectoides (Svrček \& Kubička) Dennis \& Itzerott: (Uzun et al., 2018a).

99. Kotlabaea deformis (P. Karst.) Svrček: (Kaya et al., 2016).

100. Lamprospora carbonicola Boud.: (Uzun et al., 2018c).

101. Lamprospora miniata De Not.: (Uzun et al., 2018c).

102. Lamprospora dictydiola Boud.: (Uzun et al., 2018c).

103. Octospora areolata (Seaver) Caillet \& Moyne: (Uzun et al., 2018c).

104. Octospora axillaris (Nees) M.M. Moser: (Uzun et al., 2018c).

105. Octospora coccinea (P. Crouan \& H. Crouan) Brumm.: (Uzun et al., 2018c).

106. Octospora excipulata (Clem.) Benkert: (Uzun et al., 2018c).
107. Octospora gemmicola Benkert: (Uzun et al., 2018c). 108. Octospora grimmiae Dennis \& Itzerott: (Kaya et al., 2019).

109. Octospora itzerottii Benkert: (Uzun and Kaya, 2017a).

110. Octospora leucoloma Hedw.: (Uzun et al., 2018c).

111. Octospora musci-muralis Graddon: (Uzun et al., 2018c).

112. Octospora orthotrichi (Cooke \& Ellis) K.B. Khare \& V.P. Tewari: (Uzun et al., 2018c).

113. Octospora polytrichi (Schumach.) Caillet \& Moyne: (Uzun et al., 2018c).

114. Octospora rustica (Velen.) J. Moravec: (Uzun et al., 2018c).

115. Pseudombrophila merdaria (Fr.) Brumm.: (Kaya and Uzun, 2015).

116. Pulvinula archeri (Berk.) Rifai: (Karacan et al., 2015).

117. Pulvinula carbonaria (Fuckel) Boud.: (Karacan et al., 2015).

118. Pulvinula johannis Lantieri: (Kaya et al., 2016).

119. Pulvinula laeterubra (Rehm) Pfister: (Karacan et al., 2015).

120. Pustularia patavina (Cooke \& Sacc.) Boud.: (Kaya and Uzun, 2015).

121. Pyronema domesticum (Sowerby) Sacc.: (Kaya et al., 2016).

122. Pyronema omphalodes (Bull.) Fuckel: (Kaya and Uzun, 2015).

123. Scutellinia trechispora (Berk. \& Broome) Lambotte: (Kaya et al., 2016).

124. Scutellinia umbrorum (Fr.) Lambotte: (Kaya, 2009).

125. Sepultariella semi-immersa (P.Karst.) Van Vooren, U.Lindem. \& Healy: (Uzun et al., 2018b).

126. Smardaea planchonis (Dunal ex Boud.) Korf \& W.Y. Zhuang: (Kaya et al., 2016).

127. Tarzetta catinus (Holmsk.) Korf \& J.K. Rogers: (Kaya et al., 2019).

128. Tarzetta cupularis (L.) Svrček: (Kaya et al., 2019).

129. Tricharina gilva (Boud. ex Cooke) Eckblad: (Kaya and Uzun, 2015).

130. Tricharina ochroleuca (Bres.) Eckblad: (Kaya et al., 2016).

131. Tricharina praecox (P. Karst.) Dennis: (Kaya et al., 2016).

132. Trichophaeopsis bicuspis (Boud.) Korf \& Erb: (Kaya et al., 2016).

Sarcoscyphaceae Le Gal ex Eckblad

133. Kompsoscypha chudei (Pat. ex Le Gal) Pfister: (Kaya and Uzun, 2018).

134. Pithya cupressina (Batsch) Fuckel: (Kaya and Uzun, 2018).

135. Pseudopithyella minuscula (Boud. \& Torrend) Seaver: (Kaya and Uzun, 2018).

Sarcosomataceae Kobayasi

136. Strobiloscypha cupressina B. Perić \& Pfister: (Kaya and Uzun, 2018).

Tuberaceae Dumort.

137. Tuber borchii Vittad.: (Kaya et al., 2019).

Sordariomycetes O.E. Erikss. \& Winka

Diaporthales Nannf.

Valsaceae Tul. \& C. Tul.

138. Valsa sordida Nitschke: (Kaya et al., 2019). 
Hypocreales Lindau

Nectriaceae Tul. \& C. Tul.

139. Dialonectria episphaeria (Tode) Cooke: (Kaya et al., 2019).

Sordariales Chadef. ex D. Hawksw. \& O.E. Erikss.

Lasiosphaeriaceae Nannf.

140. Lasiosphaeris hirsuta (Fr.) A.N. Mill. \& Huhndorf: (Kaya and Uzun, 2018).

Xylariales Nannf.

Diatrypaceae Nitschke

141. Diatrype bullata (Hoffm.) Fr.: (Kaya et al., 2019).

142. Diatrype disciformis (Hoffm.) Fr.: (Kaya et al., 2019).

143. Diatrype stigma (Hoffm.) Fr.: (Kaya et al., 2019).

Xylariaceae Tul. \& C. Tul.

144. Hypoxylon rubiginosum (Pers.) Fr.: (Uzun et al., 2015a).

145. Xylaria hypoxylon (L.) Grev.: (Uzun et al., 2015a).

Basidiomycota R.T. Moore

Agaricomycetes Doweld

Agaricales Underw.

Agaricaceae Chevall.

146. Agaricus arvensis Schaeff.: (Kaya, 2009).

147. Agaricus bitorquis (Quél.) Sacc.: (Kaya et al., 2019).

148. Agaricus campestris L.: (Kaya et al., 2019).

149. Agaricus moelleri Wasser: (Kaya et al., 2019).

150. Agaricus pseudopratensis (Bohus) Wasser: (Kaya 2009).

151. Agaricus sylvaticus Schaeff.: (Kaya et al., 2019).

152. Agaricus xanthodermus Genev.: (Kaya et al., 2019).

153. Battarrea phalloides (Dicks.) Pers.: (Kaya et al., 2019).

154. Bovista aestivalis (Bonord.) Demoulin: (Kaya et al., 2019).

155. Bovista nigrescens Pers.: (Kaya et al., 2019).

156. Bovista plumbea Pers.: (Kaya et al., 2019).

157. Coprinus comatus (O.F. Müll.) Pers.: (Kaya et al., 2019).

158. Crucibulum laeve (Huds.) Kambly: (Kaya et al., 2019).

159. Cyathus olla (Batsch) Pers.: (Kaya et al., 2019).

160. Cyathus stercoreus (Schwein.) De Toni: (Kaya et al., 2019).

161. Cystoderma amianthinum (Scop.) Fayod: (Kaya et al., 2019).

162. Cystodermella cinnabarina (Alb. \& Schwein.) Harmaja: (Kaya et al., 2019).

163. Echinoderma jacobi (Vellinga \& Knudsen) Gminder: (Kaya 2009).

164. Lepiota cristata (Bolton) P. Kumm.: (Uzun et al., 2015a).

165. Leucoagaricus erioderma (Malençon) Bon: (Kaya, 2009).

166. Leucoagaricus leucothites (Vittad.) Wasser: (Kaya et al., 2019).

167. Leucoagaricus serenus (Fr.) Bon \& Boiffard: (Kaya, 2009).

168. Leucocoprinus badhamii (Berk. \& Broome) Locq.: (Kaya 2009).

169. Lycoperdon excipuliforme (Scop.) Pers.: (Kaya et al., 2019).

170. Lycoperdon molle Pers.: (Kaya et al., 2019).

171. Lycoperdon nigrescens Pers.: (Kaya, 2009).
172. Lycoperdon perlatum Pers.: (Kaya et al., 2019).

173. Lycoperdon pratense Pers.: (Kaya et al., 2019).

174. Lycoperdon utriforme Bull.: (Uzun et al., 2015a).

175. Macrolepiota excoriata (Schaeff.) Wasser: (Kaya et al., 2019).

176. Macrolepiota mastoidea (Fr.) Singer: (Kaya et al., 2019).

177. Macrolepiota procera (Scop.) Singer: (Kaya et al., 2019).

178. Tulostoma brumale Pers.: (Kaya et al., 2019).

179. Tulostoma fimbriatum Fr.: (Kaya et al., 2019).

180. Tulostoma melanocyclum Bres.: (Kaya et al., 2019).

Amanitaceae R. Heim ex Pouzar

181. Amanita excelsa (Fr.) Bertill.: (Kaya et al., 2019).

182. Amanita gemmata (Fr.) Bertill.: (Uzun et al., 2015a).

183. Amanita muscaria (L.) Lam.: (Uzun et al., 2015a). 184. Amanita pantherina (DC.) Krombh.: (Uzun et al., 2015a).

185. Amanita rubescens Pers.: (Uzun et al., 2015a).

186. Amanita vaginata (Bull.) Lam.: (Kaya, 2009).

Bolbitiaceae Singer

187. Bolbitius titubans (Bull.) Fr.: (Kaya et al., 2019).

188. Conocybe apala (Fr.) Arnolds: (Kaya et al., 2019).

189. Conocybe deliquescens Huskn. \& Krisai: (Kaya et al., 2014).

190. Conocybe filaris (Fr.) Kühner: (Kaya et al., 2019).

191. Conocybe rickenii (Jul. Schäff.) Kühner: (Kaya et al., 2019).

192. Galeropsis desertorum Velen. \& Dvořák: (Kaya et al., 2019).

Chromocyphellaceae Knudsen

193. Chromocyphella muscicola (Fr.) Donk: (Uzun et al., 2017c).

Cortinariaceae R. Heim ex Pouzar

194. Cortinarius trivialis J.E. Lange: (Uzun et al., 2015a).

195. Cortinarius turgidus Fr.: (Kaya 2009).

196. Hebeloma crustuliniforme (Bull.) Quél.: (Uzun et al., 2015a).

197. Hebeloma sinapizans (Paulet) Gillet: (Kaya, 2009).

Cyphellaceae Lotsy

198. Chondrostereum purpureum (Pers.) Pouzar: (Kaya et al., 2019).

Entolomataceae Kotl. \& Pouzar

199. Entoloma rusticoides (Gillet) Noordel.: (Kaya et al., 2019).

200. Entoloma sinuatum (Bull.) P. Kumm.: (Uzun et al., 2015a).

Hydnangiaceae Gäum. \& C.W. Dodge

201. Laccaria laccata (Scop.) Cooke: (Kaya et al., 2019).

Hygrophoraceae Lotsy

202. Ampulloclitocybe clavipes (Pers.) Redhead, Lutzoni, Moncalvo \& Vilgalys: (Kaya et al., 2019).

203. Hygrocybe cantharellus (Fr.) Murrill: (Kaya, 2009).

204. Hygrocybe conica (Schaeff.) P. Kumm.: (Kaya et al., 2019).

205. Hygrophorus agathosmus (Fr.) Fr.: (Kaya, 2009).

206. Hygrophorus camarophyllus (Alb. \& Schwein.) Dumée, Grandjean \& Maire: (Kaya, 2009).

207. Hygrophorus chrysodon (Batsch) Fr.: (Uzun et al., 2015a)

208. Hygrophorus discoxanthus (Fr.) Rea: (Kaya, 2009). 
209. Hygrophorus eburneus (Bull.) Fr.: (Uzun et al., 2015a).

210. Hygrophorus purpurascens (Alb. \& Schwein.) Fr.: (Uzun et al., 2015a).

Hymenogastraceae Vittad.

211. Galerina graminea (Velen.) Kühner: (Kaya et al., 2019).

212. Galerina marginata (Batsch) Kühner: (Kaya et al., 2019).

\section{Incertae Sedis}

213. Panaeolina foenisecii (Pers.) Maire: (Kaya et al., 2019).

214. Panaeolus ater (J.E. Lange) Kühner \& Romagn. ex Bon: (Kaya et al., 2012).

215. Panaeolus fimicola (Pers.) Gillet: (Kaya et al., 2019).

216. Panaeolus olivaceus F.H. Møller: (Kaya et al., 2019).

217. Panaeolus papilionaceus (Bull.) Quél.: (Kaya et al., 2014).

Inocybaceae Jülich

218. Crepidotus pallidus (Berk. \& Broome) Knudsen: (Uzun et al. 2017b).

219. Crepidotus variabilis (Pers.) P. Kumm.: (Uzun et al., 2015a).

220. Inocybe amblyospora Kühner: (Kaya et al., 2019).

221. Inocybe bongardii (Weinm.) Quél.: (Kaya et al., 2019).

222. Inocybe cincinnata (Fr.) Quél.: (Kaya et al., 2019).

223. Inocybe geophylla (Bull.) P. Kumm.: (Kaya et al., 2019).

224. Inocybe perbrevis (Weinm.) Gillet: (Kaya 2009).

225. Inocybe pusio P. Karst.: (Kaya 2009).

226. Inocybe rimosa (Bull.) P. Kumm.: (Kaya et al., 2019).

227. Phaeomarasmius erinaceus (Fr.) Scherff. ex Romagn.: (Kaya et al., 2019).

Lyophyllaceae Jülich

228. Lyophyllum decastes (Fr.) Singer: (Uzun et al., 2015a).

Marasmiaceae Roze ex Kühner

229. Calyptella capula (Holmsk.) Quél.: (Kaya et al., 2019).

230. Henningsomyces candidus (Pers.) Kuntze: (Kaya et al., 2019).

231. Macrocystidia cucumis (Pers.) Joss.: (Kaya et al., 2019).

232. Marasmius anomalus Lasch ex Rabenh.: (Kaya et al., 2019).

233. Marasmius chordalis Fr.: (Kaya, 2009).

234. Marasmius epodius Bres.: (Kaya et al., 2019).

235. Marasmius oreades (Bolton) Fr.: (Kaya et al., 2019).

236. Marasmius wynneae Berk. \& Broome: (Kaya et al., 2019).

Mycenaceae Roze

237. Hemimycena lactea (Pers.) Singer: (Kaya et al., 2019).

238. Mycena crocata (Schrad.) P. Kumm.: (Kaya et al., 2019).

239. Mycena haematopus (Pers.) P. Kumm.: (Uzun et al., 2015a).

240. Mycena inclinata (Fr.) Quél.: (Kaya 2009).

241. Mycena meliigena (Berk. \& Cooke) Sacc.: (Uzun et al., 2017b).

242. Mycena pura (Pers.) P. Kumm.: (Kaya et al., 2019).

243. Mycena rosea Gramberg: (Kaya et al., 2019).

244. Mycena seynii Quél.: (Kaya et al., 2019).

245. Scytinotus violaceofulvus (Batsch) Courtec.: (Kaya et al., 2019).

246. Xeromphalina campanella (Batsch) Kühner \&

Maire: (Kaya et al., 2019).

247. Xeromphalina cauticinalis (With.) Kühner \& Maire: (Kaya et al., 2019).

Niaceae Jülich

248. Cyphellopsis anomala (Pers.) Donk: (Kaya et al., 2014).

249. Flagelloscypha minutissima (Burt) Donk: (Kaya et al., 2014).

250. Lachnella alboviolascens (Alb. \& Schwein.) Fr.: (Kaya et al., 2019).

251. Lachnella villosa (Pers.) Donk: (Uzun et al., 2017b).

Omphalotaceae Bresinsky

252. Gymnopus dryophilus (Bull.) Murrill: (Kaya et al., 2019).

253. Gymnopus erythropus (Pers.) Antonín, Halling \& Noordel.: (Kaya et al., 2019).

254. Gymnopus ocior (Pers.) Antonín \& Noordel.: (Kaya et al., 2019).

255. Gymnopus quercophilus (Pouzar) Antonín \& Noordel.: (Kaya et al., 2019).

256. Omphalotus olearius (DC.) Singer: (Kaya et al., 2019).

257. Omphalotus olivascens H.E. Bigelow, O.K. Mill. \& Thiers: (Kaya et al., 2014).

Physalacriaceae Corner

258. Armillaria borealis Marxm. \& Korhonen: (Kaya et al., 2019).

259. Armillaria mellea (Vahl) P. Kumm.: (Kaya et al., 2019).

260. Armillaria ostoyae (Romagn.) Herink: (Kaya 2009).

261. Cryptomarasmius corbariensis (Roum.) T.S. Jenkinson \& Desjardin: (Uzun et al., 2017b).

262. Hymenopellis radicata (Relhan) R.H. Petersen: (Uzun et al., 2015a).

263. Strobilurus stephanocystis (Kühner \& Romagn. ex Hora) Singer: (Kaya et al., 2019).

264. Strobilurus tenacellus (Pers.) Singer: (Kaya et al., 2019).

265. Xerula pudens (Pers.) Singer: (Uzun et al., 2015a).

Pleurotaceae Kühner

266. Hohenbuehelia petaloides (Bull.) Schulzer: (Kaya et al., 2019).

267. Pleurotus dryinus (Pers.) P. Kumm.: (Kaya et al., 2019).

268. Pleurotus eryngii (DC.) Quél.: (Kaya, 2009).

269. Pleurotus ostreatus (Jacq.) P. Kumm.: (Kaya et al., 2019).

Pluteaceae Kotl. \& Pouzar

270. Pluteus nanus (Pers.) P. Kumm.: (Kaya 2009).

271. Pluteus romellii (Britzelm.) Sacc.: (Kaya et al., 2019).

272. Volvopluteus gloiocephalus (DC.) Justo: (Kaya et al., 2019).

Psathyrellaceae Vilgalys, Moncalvo \& Redhead

273. Coprinellus disseminatus (Pers.) J.E. Lange: (Kaya et al., 2019).

274. Coprinellus ephemerus (Bull.) Redhead, Vilgalys \& 
Moncalvo: (Kaya et al., 2019).

275. Coprinellus impatiens (Fr.) J.E. Lange: (Kaya, 2009).

276. Coprinellus micaceus (Bull.) Vilgalys, Hopple \& Jacq. Johnson: (Kaya et al., 2019).

277. Coprinopsis atramentaria (Bull.) Redhead, Vilgalys \& Moncalvo: (Kaya et al., 2019).

278. Coprinopsis lagopides (P. Karst.) Redhead, Vilgalys \& Moncalvo: (Kaya et al., 2019).

279. Coprinopsis nivea (Pers.) Redhead, Vilgalys \& Moncalvo: (Kaya et al., 2019).

280. Lacrymaria lacrymabunda (Bull.) Pat.: (Kaya, 2009).

281. Parasola auricoma (Pat.) Redhead, Vilgalys \& Hopple: (Kaya et al., 2014).

282. Parasola plicatilis (Curtis) Redhead, Vilgalys \& Hopple: (Kaya et al., 2019).

283. Psathyrella bipellis (Quél.) A.H. Sm.: (Kaya et al., 2019).

284. Psathyrella candolleana (Fr.) Maire: (Kaya et al., 2019).

285. Psathyrella lutensis (Romagn.) Bon: (Kaya et al., 2012).

\section{Pterulaceae Corner}

286. Pterula multifida (Chevall.) Fr.: (Kaya et al., 2019).

Schizophyllaceae Quél.

287. Schizophyllum amplum (Lév.) Nakasone: (Kaya et al., 2019).

288. Schizophyllum commune Fr.: (Kaya et al., 2019).

Strophariaceae Singer \& A.H. Sm.

289. Agrocybe molesta (Lasch) Singer: (Kaya et al., 2019).

290. Agrocybe pediades (Fr.) Fayod: (Kaya et al., 2019).

291. Agrocybe praecox (Pers.) Fayod: (Kaya et al., 2014).

292. Agrocybe vervacti (Fr.) Singer: (Kaya et al., 2019).

293. Cyclocybe cylindracea (DC.) Vizzini \& Angelini: (Kaya et al., 2019).

294. Hypholoma fasciculare (Huds.) P. Kumm.: (Kaya et al., 2019).

295. Protostropharia semiglobata (Batsch) Redhead, Moncalvo \& Vilgalys: (Kaya et al., 2019).

296. Psilocybe coprophila (Bull. : Fr.) P. Kumm: (Kaya et al., 2019).

297. Stropharia aeruginosa (Curtis) Quél.: (Kaya et al., 2019).

298. Stropharia coronilla (Bull.) Quél.: (Kaya et al., 2019).

Tricholomataceae R. Heim ex Pouzar

299. Arrhenia lilacinicolor (Bon) P.-A. Moreau \& Courtec.: (Uzun et al., 2018a).

300. Arrhenia retiruga (Bull.) Redhead: (Kaya et al., 2019).

301. Arrhenia rickenii (Hora) Watling: (Kaya et al., 2019).

302. Arrhenia spathulata (Fr.) Redhead: (Kaya et al., 2019).

303. Cellypha goldbachii (Weinm.) Donk: (Uzun et al., 2018a).

304. Clitopaxillus alexandri (Gillet) G. Moreno, Vizzini, Consiglio \& P. Alvarado: (Kaya et al., 2019).

305. Clitocybe gibba (Pers.) P. Kumm.: (Kaya et al., 2019).

306. Clitocybe nebularis (Batsch) P. Kumm.: (Uzun et al., 2015a).
307. Clitocybe odora (Bull.) P. Kumm.: (Uzun et al., 2015a).

308. Clitocybe phyllophila (Pers.) P. Kumm.: (Kaya, 2009).

309. Cotylidia diaphana (Cooke) Lentz: (Kaya, 2009).

310. Infundibulicybe geotropa (Bull.) Harmaja: (Kaya et al., 2019).

311. Lepista nuda (Bull.) Cooke: (Kaya et al., 2019).

312. Lepista sordida (Schumach.) Singer: (Uzun et al. 2015a).

313. Leucopaxillus gentianeus (Quél.) Kotl.: (Kaya et al., 2019).

314. Melanoleuca cognata (Fr.) Konrad \& Maubl.: (Kaya et al., 2019).

315. Melanoleuca exscissa (Fr.) Singer: (Kaya et al., 2019).

316. Melanoleuca polioleuca (Fr.) Kühner \& Maire: (Kaya et al., 2019).

317. Melanoleuca stridula (Fr.) Singer: (Kaya et al., 2019).

318. Myxomphalia maura (Fr.) H.E. Bigelow: (Kaya et al., 2019).

319. Pseudoclitocybe cyathiformis (Bull.: Fr.) Singer: (Kaya et al., 2019).

320. Resupinatus taxi (Lév.) Thorn, Moncalvo \& Redhead: (Uzun et al., 2018a).

321. Resupinatus trichotis (Pers.) Singer: (Kaya et al., 2019).

322. Rimbachia neckerae (Fr.) Redhead: (Uzun et al., 2018a).

323. Tricholoma anatolicum H.H. Doğan \& Intini: (Uzun et al., 2015a).

324. Tricholoma batschii Gulden: (Kaya, 2009; Uzun et al., 2015a).

325. Tricholoma equestre (L.) P. Kumm.: (Uzun et al., 2015a).

326. Tricholoma terreum (Schaeff.) P. Kumm.: (Kaya et al., 2019).

327. Tricholoma virgatum (Fr.) P. Kumm.: (Kaya, 2009).

Tubariaceae Vizzini

328. Tubaria conspersa (Pers.) Fayod: (Kaya et al., 2019).

Typhulaceae Jülich

329. Typhula fistulosa (Holmsk.) Olariaga: (Kaya et al., 2019).

330. Typhula setipes (Grev.) Berthier: (Uzun et al., 2017b)

Auriculariales J. Schröt.

Auriculariaceae Fr.

331. Exidia glandulosa (Bull.) Fr.: (Kaya et al., 2019).

332. Exidia nigricans (With.) P. Roberts: (Kaya et al., 2019).

Boletales E.-J. Gilbert

Boletaceae Chevall.

333. Boletus edulis Bull.: (Kaya et al., 2019).

334. Boletus erythropus Pers.: (Kaya et al., 2019).

335. Boletus reticulatus Schaeff.: (Kaya, 2009).

336. Xerocomellus chrysenteron (Bull.) Šutara: (Kaya et al., 2019).

337. Xerocomellus porosporus (Imler ex Watling) Šutara: (Kaya, 2009).

Diplocystidiaceae Kreisel

338. Astraeus hygrometricus (Pers.) Morgan: (Kaya et al., 2019). 
Gomphidiaceae Maire ex Jülich

339. Chroogomphus rutilus (Schaeff.) O.K. Mill.: (Kaya et al., 2019).

Paxillaceae Lotsy

340. Melanogaster ambiguus (Vittad.) Tul. \& C. Tul.: (Uzun et al., 2015a).

341. Melanogaster broomeanus Berk.: (Uzun et al., 2015a).

Rhizopogonaceae Gäum. \& C.W. Dodge

342. Rhizopogon luteolus Fr.: (Kaya et al., 2019).

343. Rhizopogon roseolus (Corda) Th. Fr.: (Kaya et al., 2019).

Sclerodermataceae Corda

344. Pisolithus arhizus (Scop.) Rauschert: (Kaya et al., 2019).

345. Scleroderma cepa Pers.: (Kaya et al., 2019).

346. Scleroderma polyrhizum (J.F. Gmel.) Pers.: (Uzun et al., 2015a).

347. Scleroderma verrucosum (Bull.) Pers.: (Kaya et al., 2019).

Suillaceae Besl \& Bresinsky

348. Suillus collinitus (Fr.) Kuntze: (Kaya et al., 2019).

349. Suillus granulatus (L.) Roussel: (Kaya et al., 2019).

350. Suillus luteus (L.) Roussel: (Kaya et al., 2019).

Tapinellaceae C. Hahn

351. Tapinella panuoides (Fr.) E.-J. Gilbert: (Kaya et al., 2019).

Cantharellales Gäum

Clavulinaceae Donk

352. Clavulina cinerea (Bull.) J. Schröt.: (Kaya et al., 2019).

353. Clavulina coralloides (L.) J. Schröt.: (Uzun et al., 2015a).

Geastrales K. Hosaka \& Castellano

Geastraceae Corda

354. Geastrum minimum Schwein.: (Kaya et al., 2019).

355. Geastrum pectinatum Pers.: (Kaya et al., 2019).

356. Geastrum rufescens Pers.: (Uzun et al., 2015a).

357. Geastrum triplex Jungh.: (Uzun et al., 2015a).

358. Sphaerobolus stellatus Tode: (Kaya et al., 2019).

Gomphales Jülich

Gomphaceae Donk

359. Gautieria monticola Harkn.: (Uzun et al., 2015a).

360. Gautieria trabutii (Chatin) Pat.: (Kaya et al., 2019).

361. Gomphus clavatus (Pers.) Gray: (Uzun et al., 2015a).

362. Ramaria flava (Schaeff.) Quél.: (Uzun et al., 2015a).

363. Ramaria stricta (Pers.) Quél.: (Kaya, 2009).

Hymenochaetales Oberw.

Hymenochaetaceae Imazeki \& Toki

364. Phellinus hartigii (Allesch. \& Schnabl) Pat.: (Kaya et al., 2019).

365. Phellinus igniarius (L.) Quél.: (Kaya et al., 2014).

366. Phellinus pomaceus (Pers.) Maire: (Kaya et al., 2019).

Hysterangiales K. Hosaka \& Castellano

Hysterangiaceae E. Fisch.

367. Hysterangium clathroides Vittad.: (Uzun et al., 2015a).

Phallales E. Fisch.

Phallaceae Corda

368. Phallus impudicus L.: (Kaya et al., 2019).
Polyporales Gäum.

Ganodermataceae Donk

369. Ganoderma adspersum (Schulzer) Donk: (Kaya et al., 2019).

370. Ganoderma applanatum (Pers.) Pat.: (Kaya et al., 2014).

371. Ganoderma lucidum (Curtis) P. Karst.: (Kaya et al., 2019).

Meruliaceae Rea

372. Abortiporus biennis (Bull.) Singer: (Kaya et al., 2014).

373. Bjerkandera adusta (Willd.) P. Karst.: (Kaya et al., 2019).

Phanerochaetaceae Jülich

374. Terana coerulea (Lam.) Kuntze: (Kaya et al., 2019).

Polyporaceae Fr. ex Corda

375. Cerioporus varius (Pers.) Zmitr. \& Kovalenko: (Kaya, 2009).

376. Fomes fomentarius (L.) Fr.: (Kaya et al., 2019).

377. Lentinus arcularius (Batsch) Zmitr.: (Kaya et al., 2019).

378. Lentinus brumalis (Pers.) Zmitr.: (Kaya et al., 2019).

379. Lentinus tigrinus (Bull.) Fr.: (Kaya et al., 2019).

380. Lenzites betulina (L.) Fr.: (Kaya et al., 2019).

381. Royoporus badius (Pers.) A.B. De: (Kaya et al., 2019).

382. Trametes hirsuta (Wulfen) Lloyd: (Kaya et al., 2019).

383. Trametes trogii Berk.: (Kaya et al., 2014).

384. Trametes versicolor (L.) Lloyd: (Kaya et al., 2019).

385. Trichaptum abietinum (Dicks.) Ryvarden: (Uzun et al., 2015a).

386. Trichaptum fuscoviolaceum (Ehrenb.) Ryvarden: (Uzun et al., 2015a).

Russulales Kreisel ex P.M. Kirk, P.F. Cannon \& J.C.

David

Auriscalpiaceae Maas Geest.

387. Lentinellus cochleatus (Pers.) P. Karst.: (Uzun et al., 2015a).

388. Lentinellus micheneri (Berk. \& M.A. Curtis) Pegler: (Kaya et al., 2019).

Hericiaceae Donk

389. Hericium abietis (Weir ex Hubert) K.A. Harrison: (Kaya, 2009).

Russulaceae Lotsy

390. Lactarius deliciosus (L.) Gray: (Kaya et al., 2019).

391. Lactarius piperatus (L.) Pers.: (Kaya, 2009).

392. Lactarius torminosus (Schaeff.) Gray: (Kaya et al., 2019).

393. Russula albonigra (Krombh.) Fr.: (Kaya, 2009).

394. Russula brevipes Peck: (Uzun et al., 2015a).

395. Russula delica Fr.: (Kaya, 2009).

396. Russula virescens (Schaeff.) Fr.: (Kaya et al., 2019). Stereaceae Pilát

397. Stereum hirsutum (Willd.) Pers.: (Kaya et al., 2019).

Bankeraceae Donk

398. Sarcodon imbricatus (L.) P. Karst.: (Kaya, 2009).

Thelephorales Corner ex Oberw.

Bankeraceae Donk

399. Hydnellum caeruleum (Hornem.) P. Karst.: (Kaya et al., 2019). 
Thelephoraceae Chevall.

400. Thelephora terrestris Ehrh.: (Kaya et al., 2019).

Dacrymycetes Doweld

Dacrymycetales Henn.

Dacrymycetaceae J. Schröt.

401. Calocera cornea (Batsch) Fr.: (Kaya et al., 2019).

402. Dacrymyces capitatus Schwein.: (Kaya et al., 2019).

403. Dacrymyces stillatus Nees: (Kaya et al., 2019).

Tremellomycetes Doweld

Tremellales Fr.

Hyaloriaceae Lindau

404. Myxarium nucleatum Wallr.: (Uzun et al., 2016).

\section{Discussions}

Compiling the overall macrofungal taxa that had been presented so far, Gaziantep was determined to hosts a total of 404 taxa within Ascomycota and Basidiomycota. One hundred and forty five (\%35.89) of them belong to Ascomycota while 259 (\%64.11) belong to Basidiomycota.

The taxa determined in the region were found to distribute in eight classes (Table 1) and 23 orders (Table 2). The most diverste class and the order were found to be Agaricomcyetes and Agaricales respectively.

Eighty macromycete families were represented in Gaziantep. Pyronemataceae, Agaricaceae, Tricholomataceae, Psathyrellaceae, and Pezizaceae are the first 5 most crowded families in the region. The most crowded genus is Octospora with 12 taxa. It was followed by Helvella, Agaricus, Inocybe and Mycena respectively.

Eighty eight of the 404 taxa are edible, but only six taxa, Agaricus campestris, Coprinus comatus, Pleurotus ostreatus, Terfezia boudieri, Tricholoma anatolicum, and Volvopluteus gloiocephalus, are collected and consumed in the region by local people. Terfezia boudieri have local economic importance while Tricholoma anatolicum have international economic importance (Kaya et al., 2019). Two hundred and eighty three of the determined taxa were regarded as inedible while 33 are more or less poisonous.

Among the determined taxa, 103 are lignicolous, 18 are coprophilous, 7 are pyrophilous, 24 are bryophilous and the rest of the taxa are terricolous. Twenty two of them were also determined as hygogeous.

The presented articles indicate that Gaziantep hosts the first locality in Turkey for 95 of the determined species. These species are Aleuria exigua, Arrhenia lilacinicolor, Ascobolus carbonarius, A. crenulatus, A. foliicola, A. immersus, Barssia hellenica Bisporella sulfurina,

Table 1. Distribution of the determined taxa in classes.

\begin{tabular}{llr}
\hline Division & Class & \# of taxa \\
\hline \multirow{4}{*}{ Ascomycota } & Pezizomycetes & 92 \\
& Leotiomycetes & 42 \\
& Sordariomycetes & 8 \\
& Orbiliomycetes & 2 \\
& Dothideomycetes & 1 \\
\hline \multirow{3}{*}{ Basidiomycota } & Agaricomycetes & 255 \\
& Dacrymycetes & 3 \\
& Tremellomycetes & 1 \\
\hline
\end{tabular}

Table 2. Distribution of the determined taxa in orders.

\begin{tabular}{llr}
\hline Division & Order & \# of taxa \\
\hline & Pezizales & 92 \\
& Helotiales & 38 \\
& Xylariales & 5 \\
Rhytismatales & 4 \\
Ascomycota & Orbiliales & 2 \\
& Diaporthales & 1 \\
& Hypocreales & 1 \\
& Patellariales & 1 \\
& Sordariales & 1 \\
\hline & Agaricales & 185 \\
& Boletales & 19 \\
& Polyporales & 18 \\
& Russulales & 11 \\
& Geastrales & 5 \\
& Gomphales & 5 \\
& Dacrymycetales & 3 \\
& Hymenochaetales & 3 \\
& Thelephorales & 3 \\
& Auriculariales & 2 \\
& Cantharellales & 2 \\
& Hysterangiales & 1 \\
& Phallales & 1 \\
& Tremellales & 1 \\
\hline & &
\end{tabular}

Calycina conorum, Cellypha goldbachii, Cheilymenia catenipila, Cheilymenia pulcherrima, C. vitellina, Chromocyphella muscicola, Coccomyces delta, C. dentatus, Cortinarius turgidus, Cotylidia diaphana, Crepidotus pallidus, Cryptomarasmius corbariensis, Cyclaneusma minus, C. niveum, Discocistella grevillei, Geopyxis majalis, Geopyxis vulcanalis, Helvella compressa, Humaria aurantia, Hyalopeziza millepunctata, Hyalorbilia inflatula, Hymenoscyphus janthinum, Hypotarzetta insignis, Hypoxylon rubiginosum, Inermisia gyalectoides, Kompsoscypha chudei, Kotlabaea deformis, Lachnella villosa, Lachnum fuscescens, Lamprospora carbonicola, L. dictydiola, L. miniata, Lasiobelonium horridulum var. capitatum, L. variegatum, Lasiobolus cuniculi, Lasiosphaeris hirsuta, Lepiota jacobi, Leucoagaricus erioderma, L. serenus, Marcelleina atroviolacea, M. rickii, Mollisia hydrophila, Mycena meliigena, Myxarium nucleatum, Neobulgaria pura, Octospora areolata, $O$. axillaris, $O$. coccinea, $O$. excipulata, O. gemmicola, O. itzerottii, O. musci-muralis, O. orthotrichi, O. polytrichi, O. rustica, Orbilia aristata, Perrotia flammea, Phaeohelotium umbilicatum, Pithya cupressina, Pseudombrophila merdaria, Pseudopithyella minuscula, Pulvinula archeri, $P$. carbonaria, $P$. johannis, P. laeterubra, Pustularia patavina, Pyronema domesticum, P. omphalodes, Resupinatus taxi, Rimbachia neckerae, Rodwayella citrinula, Saccobolus glaber, Sclerotinia trifoliorum, Scutellinia trechispora, Sepultariella semi-immersa, Smardaea planchonis, Strobiloscypha cupressina, Tapesia strobilicola, Thecotheus holmskioldii, T. pelletieri, Tricharina gilva, T. ochroleuca, T. praecox, Trichobelonium kneiffii, Trichopeziza subsulphurea, Trichophaeopsis bicuspis and Typhula setipes. 


\section{References}

Acar İ, Uzun Y (2017). An interesting half-free morel record for Turkish Mycobiota (Morchella populiphila M. Kuo, M.C. Carter \& J.D. Moore). The Journal of Fungus 8(2): 125-128.

Acar İ, Uzun Y, Keleş A, Dizkırcı A (2019). Suillellus amygdalinus, a new species record for Turkey from Hakkari Province . Anatolian Journal of Botany 3(1): 25-27.

Akata I, Doğan HH, Öztürk Ö, Bozok F (2018). Suillus lakei, an interesting record for Turkish Mycobiota. The Journal of Fungus 9(2): 110-116.

Akçay ME (2019). A new edible macrofungus record for Turkey. Journal of Natural \& Applied Sciences of East 2(1): 10-15.

Akçay ME (2020). A new record for the Mycota of Turkey. Anatolian Journal of Botany 4(1): 8-10.

Çolak ÖF, Kaygusuz O, Battistin E (2017). Paralepistopsis amoenolens: First record of a rare and poisonous taxon in Turkey. Turkish Journal of Life Sciences 2(2): 175-179.

Demirel GN, Allı H (2019). Macrofungi Determined in Köyceğiz (Muğla) District. The Journal of Fungus 10(2): 133-142.

Demirel K, Koçak MZ (2016). Zilan Vadisi’nin (Erciş-VAN) Makrofungal Çeşitliliği. Mantar Dergisi 7(2): 122-134.

Demirel K, Uzun Y, Keleş A, Akçay ME, Acar İ (2017). Macrofungi of Karagöl-Sahara National Park (Artvin/Turkey). Biological Diversity and Conservation 10(2): 32-40.

Doğan HH, Bozok F, Taşkın H (2018). A new species of Barssia (Ascomycota, Helvellaceae) from Turkey. Turkish Journal of Botany 42(5): 636-643.

Güngör H, Solak MH, Allı H, Işı1loğlu M, Kalmış E (2016). Contributions to the macrofungal diversity of Hatay province, Turkey. Biological Diversity and Conservation 9(1): 101-106.

Index Fungorum. (2019). http://www.indexfungorum.org/names/Names.asp / [20 December 2019].

Intini M, Doğan HH, Riva A (2003). Tricholoma anatolicum spec. nov.: A new member of the Matsutake group. Micologia e Vegetazione Mediterranea 18(2): 135-142.

Iş1k H (2020). Agaricus, Steccherinum, and Typhula species new for Turkey. Mycotaxon 135: 213-222.

Işık H, Türkekul İ (2018). Tokat’tan yeni bir lignikol mantar kaydı: Lachnum subvirgineum Baral. Kahramanmaraş Sütçü İmam Üniversitesi Tarım ve Doğa Dergisi 21(4): 555-558.

Iş1loğlu M, Allı H, Solak MH, Watling R (2009). A new Marasmius on Castanea sativa from Turkey. Mycotaxon 107: 343 -347.

Işılloğlu M, Allı H, Spooner BM, Solak MH (2010). Morchella anatolica (Ascomycota), a new species from southwestern Anatolia, Turkey. Mycologia 102 (2): 455-458.

Karacan İH, Uzun Y, Kaya A, Yakar S (2015). Pulvinula Boud., a new genus and three pulvinuloid macrofungi taxa new for Turkey. Biological Diversity and Conservation 8(2): 161-164.

Kaşık G, Aktaş S, Alkan S, Öztürk C (2017). Selçuk Üniversitesi Alaeddin Keykubat Kampüsü (Konya) Mantarlarına İlaveler. Mantar Dergisi 8(2): 129-136.

Kaya A (2009). Macrofungi of Huzurlu High Plateau (Gaziantep-Turkey). Turkish Journal of Botany 33(6): 429-437.

Kaya A, Demirel K, Uzun Y (2012). Macrofungal diversity of Araban (Gaziantep/Turkey) district. Biological Diversity and Conservation 5(3): 162-166.

Kaya A, Karacan İH, Uzun Y (2015). Three Phragmites Adans. inhabiting fungi, new for Turkey. Biological Diversity and Conservation 8(1): 143-146.

Kaya A, Kaya ÖF, Uzun Y, Karacan İH (2014). Macromycetes of Yavuzeli and Şehitkâmil (Gaziantep) Districts. Biological Diversity and Conservation 7(3): 138-142.

Kaya A, Uzun Y (2015). Six new genus records for Turkish Pezizales from Gaziantep province. Turkish Journal of Botany 39(3): 506-511.

Kaya A, Uzun Y (2018). New Contributions to the Turkish Ascomycota. Turkish Journal of Botany 42(5): 644-652.

Kaya A, Uzun Y, Karacan İH, Yakar S (2016). Contributions to Turkish Pyronemataceae from Gaziantep province. Turkish Journal of Botany 40(3): 298-307.

Kaya A, Uzun Y, Karacan İH, Yakar S (2018). New additions to Turkish Helotiales and Orbiliales. Kastamonu Üniv. Journal of Forestry Faculty 18(1): 46-52.

Kaya A, Uzun Y, Karacan İH, Yakar S (2019). Contributions to the Macromycota of Gaziantep Province. Kastamonu Univ. Journal of Forestry Faculty 19(3): 329-341.

Kaygusuz O, Knudsen H, Türkekul İ, Çolak ÖF (2020). Volvariella turcica, a new species from Turkey, and a multigene phylogeny of Volvariella. Mycologia 112(3): 577-587.

Keleş A (2019a). New records of macrofungi from Trabzon province (Turkey). Applied Ecology and Environmental Research 17(1): 1061-1069.

Keleş A (2019b). New records of Hymenoscyphus, Parascutellinia, and Scutellinia for Turkey. Mycotaxon 134(1): 169-175.

Kirk PM, Ansell AE (1992). Authors of fungal names. International Mycological Institute, CABI, Wallingford. 
Kirk PM, Cannon PF, David JC, Stalfers JA (2004). Authors of fungal names. CABI Bioscience, Wallingford. Electronic version: http:// www. indexfungorum. org/ Names /Names.asp / [20 December 2019].

Kirk PM, Cannon PF, Minter DW, Stalpers JA (2008). Dictionary of the Fungi. 10th ed. Wallingford, UK: CAB International.

Sadullahoğlu C, Uzun Y (2020). Karz Dağı (Tatva-Bitlis) ve çevresinde belirlenen makrofunguslar. Mantar Dergisi 11(1): 1-11.

Sesli E, Antonin V, Contu M (2018). A new species of Hygrophorus, H. yadigarii sp. nov. (Hygrophoraceae), with an isolated systematic position within the genus from the Colchic part of Turkey. Turkish Journal of Botany 42(2): 224-232.

Sesli E, Antonín V, Hughes KW (2017). Marasmiellus istanbulensis (Omphalotaceae), a new species from Belgrade Forest (İstanbulTurkey). Plant Biosystems -An International Journal Dealing with all Aspects of Plant Biology, DOI: 10.1080/11263504.2017.1317670

Sesli E, Denchev CM (2014). Checklists of the myxomycetes, larger ascomycetes, and larger basidiomycetes in Turkey. 6th edn. Mycotaxon Checklists Online (http,//www.mycotaxon.com/resources/checklists/sesli-v106-checklist.pdf): 1-136.

Sesli E, Vizzini A (2017). Two new Rhodocybe species (sect. Rufobrunnea, Entolomataceae) from the East Black Sea coast of Turkey. Turkish Journal of Botany 41(2): 200-210.

Sesli E, Vizzini A, Contu M (2015). Lyophyllum turcicum (Agaricomycetes: Lyophyllaceae), a new species from Turkey. Turkish Journal of Botany 39(3): 512-519.

Solak MH, Işıloğlu M, Kalmış E, Allı H (2015). Macrofungi of Turkey, Checklist. Volume-II. Bornova: Üniversiteliler Ofset.

Şen İ, Allı H (2019). Tricholoma (Fr.) Staude in Aegean region of Turkey. Turkish Journal of Botany 43(6): 817-830.

Taşkın H, Doğan HH, Büyükalaca S, Clowez P, Moreau PA, O’Donnell K (2016). Four new morel (Morchella) species in the elata subclade (M. sect. Distantes) from Turkey. Mycotaxon 131(2): 467-482.

Topçu Sesli A, Sesli E (2017). Cuphophyllus flavipes (Britzelm.) Bon (Hygrophoraceae): Türkiye için yeni bir şapkalı mantar. Bağbahçe Bilim Dergisi 4(2): 8-12.

Türkekul İ, Işı1k H (2017). Bozatalan (Tokat) Yöresi Makrofungusları. Kafkas Üniversitesi Fen Bilimleri Enstitüsü Dergisi 9(1): 511.

Türkekul İ, Işık H (2019). Macrofungal Biodiversity of Reşadiye(Tokat) District. Acta Biologica Turcica 32(2):95-101.

Uzun Y, Acar İ, Akçay ME, Kaya A (2017). Contributions to the macrofungi of Bingöl, Turkey. Turkish Journal of Botany 41(5): 516-534.

Uzun Y, Acar İ, Akçay ME, Sadullahoğlu C (2020). Kağızman (Kars) Yöresi Makrofungusları. Mantar Dergisi 11(1): 19-28.

Uzun Y, Karacan İH, Yakar S, Kaya A (2017a). Octospora Hedw., A New Genus Record for Turkish Pyronemataceae. Anatolian Journal of Botany 1(1): 18-20.

Uzun Y, Karacan İH, Yakar S, Kaya A (2018a). New Additions to Turkish Tricholomataceae. Anatolian Journal of Botany 2(2): 65-69.

Uzun Y, Karacan İH, Yakar S, Kaya A (2018c). New bryophillic Pyronemataceae records for Turkish Pezizales from Gaziantep province. Anatolian Journal of Botany 2(1): 28-38.

Uzun Y, Kaya A, Karacan İH, Kaya ÖF, Yakar S (2015a). Macromycetes determined in Islahiye (Gaziantep/Turkey) district. Biological Diversity and Conservation 8(3): 209-217.

Uzun Y, Kaya A, Karacan İH, Kaya ÖF, Yakar S (2015b). Neobulgaria Petr. and Trichopeziza Fuckel, Two New Genus Record for Turkish Lachnaceae. Mantar Dergisi 6(2): 58-61.

Uzun Y, Kaya A, Karacan İH, Yakar S (2017b). New additions to Turkish Agaricales. Biological Diversity and Conservation 10(2): 8-13.

Uzun Y, Kaya A, Karacan İH, Yakar S. (2017c). New additions to Turkish Hyaloscyphaceae. The Journal of Fungus 8(1): 13-19.

Uzun Y, Kaya A, Yakar S, Karacan İH (2016). Hyaloriaceae Lindau, A New Family Record for Turkish Mycobiota. Mantar Dergisi 7(1): 24-28.

Uzun Y, Yakar S, Karacan İH, Kaya A (2018b). New additions to the Turkish Pezizales. Turkish Journal of Botany 42(3): 335345.

Vizzini A, Antonin V, Sesli E, Contu M (2015). Gymnopus trabzonensis sp. nov. (Omphalotaceae) and Tricholoma virgatum var. fulvoumbonatum var. nov. (Tricholomataceae), two new white-spored agarics from Turkey. Phytotaxa 226 (2): 119-130.

Vizzini A, Baroni TJ, Sesli E, Antonín V, Saar I (2016). Rhodocybe tugrulii (Agaricales, Entolomataceae), a new species from Turkey and Estonia based on morphological and molecular data, and a new combination in Clitocella (Entolomataceae). Phytotaxa 267(1): 1-15.

Watling R, Işıloglu M, Baş Sermenli H (2010). Observations on the Bolbitiaceae 31. Conocybe volviradicata sp. nov. Mycotaxon 114: 145-149.

Yıldız MS, Türkekul İ, Işık H (2019). Macrofungal biodiversity of Pazar (Tokat) district. BEÜ Journal of Science 8(2): $387-395$. 